\title{
Vibrational analysis of hydroxyacetone
}

Vlasta Mohaček-Grošev ${ }^{\mathrm{a}}$

\begin{abstract}
In order to be able to fully understand the vibrational dynamics of monosaccharide sugars, we started with hydroxyacetone $\mathrm{CH}_{2} \mathrm{OHCOCH}_{3}$, and glycolaldehyde $\mathrm{CH}_{2} \mathrm{OHCOH}$, which are among the smallest molecules that contain hydroxyl and carbonyl group on neighboring carbon atoms. This sterical configuration is characteristic for saccharides and determines their biochemical activity. In this work vibrational analysis of hydroxyacetone was undertaken by performing the normal coordinate analysis for glycolaldehyde first, and transferring these force constants to hydroxyacetone. The observed Raman and infrared bands for $90 \mathrm{wt} \%$ solution of hydroxyacetone in water (acetol) were used as a first approximation for the bands of free hydroxyacetone. The number of observed Raman and infrared bands for acetol exceeds the number of calculated values for the most stable hydroxyacetone conformer with $\mathrm{C}_{\mathrm{s}}$ symmetry, which suggests more than one conformer of hydroxyacetone in water solution. In particular, there are two bands both in infrared (1083 and $\left.1057 \mathrm{~cm}^{-1}\right)$ and in Raman spectrum (1086.5 and $1053 \mathrm{~cm}^{-1}$ ) that are assigned to the $\mathrm{CO}$ stretching mode and this is one of the indicators of several hydroxyacetone conformers in the solution. Additional information was obtained from low temperature Raman spectra: at $240 \mathrm{~K}$ a broad assymmetric band centered around $280 \mathrm{~cm}^{-1}$ appears, suggesting a disorder in the orientation of hydroxyl groups. Glassy state forms at $\sim 150 \mathrm{~K}$. The broad band at $80 \mathrm{~cm}^{-1}$ is assigned to frozen torsions of hydroxymethyl groups.
\end{abstract}

\section{PACS}

31.50 Bc; $33.15 \mathrm{Bh} ; 33.20 \mathrm{Ea} ; 33.20 \mathrm{Fb}$

\section{Introduction}

Our knowledge of cellular molecular organization has advanced rapidly in recent years [1] and [2]. In particular, carbohydrates gained on significance since not only they serve as energy storage, participate in the building of DNA and RNA and are structural elements in plants' cell walls [3], but also bind with proteins to form glycoproteins and proteoglycans and thus influence cell growth and maturization of specialized tissue. They also form sophisticated extracellular matrices throughout the organism [4] and [5].

\footnotetext{
${ }^{a}$ Materials Physics Department, Ruđer Bošković Institute, Bijenička cesta 54, POB 180, 10002 Zagreb, Croatia (mohacek@irb.hr)
} 
Glycolaldehyde together with smaller sugars is produced at the initial stages of nonenzymatic glycosylation of proteins, the pathological reaction which produces cross-linking and disables proteins' biological function [6]. It is known that the advanced glycation end products (AGEs) agglomerate in the tissue affected by Diabetes mellitus, such as in eye lens [7] and [8].

Common to all monosaccharides, be they aldehydes or ketones, is the occurrence of carbonyl group next to hydroxyl group in the chain conformation of sugar molecule. Since the behavior of these hydroxy-carbonyl compounds in water is extremely complex (for glycolaldehyde and glyceraldehyde see [9], [10] and [11]), vibrational analysis of solutions cannot be reliable unless the spectral database of the participating chemical species is complete. As a first step glycolaldehyde $\mathrm{CH}_{2} \mathrm{OHCOH}$, and hydroxyacetone $\mathrm{CH}_{2} \mathrm{OHCOCH}_{3}$ were chosen for study.

Glycolaldehyde at room temperature appears in the form of a dimer (2,5-dihydroxy-1,4-dioxane) which crystalizes in a mixture of two phases, $\alpha$ and $\beta$, as found by Kobayashi et al. [12]. The symmetry of these crystal phases as well as molecular conformation in each of them has not been determined yet by $\mathrm{X}$ ray crystallography, but the infrared and Raman study of Kobayashi et al. [12] suggests the trans axial position of two $\mathrm{OH}$ groups in the phase $\alpha$, and their equatorial position in the phase $\beta$. Previous research on glycolaldehyde that employed vibrational sectroscopy includes the work of Michelsen and Klaboe [13], infrared matrix isolation study of Aspiala et al. [14], and, more recently, Yaylayan et al. studied transformation of glycolaldehyde with temperature and in different solvents [15]. Quite a few ${ }^{1}$ HNMR studies were done on glycolaldehyde [9], [10], [11], [16], [17], [18] and [19] and one on hydroxyacetone in aqueous solutions [20]. Whereas glycolaldehyde exhibits really complicated behavior in water, methanol and dimethyl sulphoxide, forming several types of open and closed dimers, as well as hydrate and enediol [10] and [15], hydroxyacetone in water seems to remain a monomer [20]. Both free glycoladehyde [21] and [22] and hydroxyacetone [23] exhibit intramolecular hydrogen bonding that establishes planar $\mathrm{C}-\mathrm{O} \ldots \mathrm{HO} \square \mathrm{C}$ conformation as the energetically most favorable.

Since there were some discrepancies in the vibrational assignment of the glycolaldehyde bands and no assignment has been done for hydroxyacetone so far, semi-empirical normal coordinate analysis was performed with valence force constants transferred from well established force fields of acetone and acetaldehyde [24] and ethylene glycol [25], with very few modifications. The purpose of this calculation in the harmonic approximation was to identify the hydroxyacetone bands most sensitive to hydrogen bonding and conformation of the molecule by comparing the calculated bands positions with the observed Raman and infrared bands of water solution of hydroxyacetone (acetol).

\section{Experimental}

Raman spectra of liquid acetol in an open glass cuvette were obtained with DILOR Z24 triple monochromator operating in sequential mode, in standard $90^{\circ}$ geometry. The step was $1 \mathrm{~cm}^{-1}$, 
the slitwidth was $300 \mu \mathrm{m}$, time constant $1 \mathrm{~s}$, and the accuracy of the observed bands positions better than $1 \mathrm{~cm}^{-1}$. The COHERENT INNOVA 100 argon ion laser operating at $514.5 \mathrm{~nm}$ with the laser power of $200 \mathrm{~mW}$ served as an excitation source. Low temperature Raman spectra of acetol were obtained while the sample, sealed under vacuum in the capillary tube, was cooled down with CTI CRYOGENICS closed cycle helium cryostat with Lake Shore controller.

Infrared spectra were recorded on Perkin Elmer 2000 instrument, with 20 scans and the resolution of $1 \mathrm{~cm}^{-1}$ using Perkin Elmer liquid cell with $\mathrm{KBr}$ windows.

Commercial acetol (90 wt.\% solution of hydroxyacetone in water) was purchased from SigmaAldrich and used without further purification. The substance turned out to be very hygroscopic, and water absorption from the air very pronounced. Therefore in several occassions we used the stream of hot air to evaporate the water from the solution, notifying the spectral changes. The temperature to which the sample was warmed up is estimated to be $\sim 50{ }^{\circ} \mathrm{C}$, which is much less than the boiling point of acetol $\left(145^{\circ} \mathrm{C}\right.$ from manufacturer's data). The vapour pressure at room temperature is estimated to be less than $0.5 \mathrm{kPa}$. An attempt to record Raman spectrum of vapour failed due to water condensation on windows of quartz Raman cell during the heating of solution.

Vibrational frequencies were calculated in harmonic approximation using program package of Schachtschneider [26].

\section{Results}

Although having similar chemical composition, glycolaldehyde and hydroxyacetone have rather different equipotential energy surface judging by the ones calculated by ab initio methods [14], [27] and [28]. We define as $\tau_{1}$ the $\mathrm{CH}_{2} \mathrm{O}$ torsional angle around $\mathrm{C}_{1}-\mathrm{C}_{2}$ bond, and as $\tau_{2}$ the $\mathrm{OH}$ torsional angle around $\mathrm{C}_{1}-\mathrm{O}_{3}$ bond. In the notation of reference [14] we denote cis conformation with respect to $\tau_{1}$ with big letter $\mathbf{C}$, and cis conformation with respect to $\tau_{2}$ with small letter $\mathbf{c}$. In the same manner trans conformers are denoted with $\mathbf{T}$ and $\mathbf{t}$. For both molecules the most stable conformer is the one where hydroxyl and carbonyl group are in cis position (as shown in Fig. 4 and Fig. 5), or Cc. Quantum calculations on glycoladehyde predict that the next

stable conformer $\mathbf{T t}$ has $\sim 10$ [14] or $14 \mathrm{~kJ} \mathrm{~mol}^{-1}$ [27] higher energy than $\mathbf{C c}$ conformer. For hydroxyacetone the minimum of the $\mathbf{C c}$ conformer is even deeper-the next stable conformation lies at least $90 \mathrm{~kJ} \mathrm{~mol}^{-1}$ higher [28].

Polarized Raman spectra of acetol are shown in Fig. 1 and Fig. 2. In Fig. 3 the infrared and Raman spectrum of the acetol previously heated for $15 \mathrm{~min}$ in the stream of hot air are compared. This warming up turned out to be a necessity for obtaining a good quality infrared spectrum since the sample sorbes water from the air rather quickly. Two spectral differences were noted in the Raman spectrum on heating: the band at $2854 \mathrm{~cm}^{-1}$ shifts to lower wavenumbers, and a 
Mohaček-Grošev, V., (2005), "Vibrational analysis of hydroxyacetone" Spectrochimica Acta Part A: Molecular and Biomolecular Spectroscopy, Vol. 61, No. 3, pp. 477-484.

shoulder at $1053 \mathrm{~cm}^{-1}$ becomes a pronounced band at $1028.5 \mathrm{~cm}^{-1}$. In Table 1 the calculated frequencies for glycolaldehyde are compared to the positions of observed bands form literature, and in Table 2 observed and calculated frequencies for hydroxyacetone are listed.

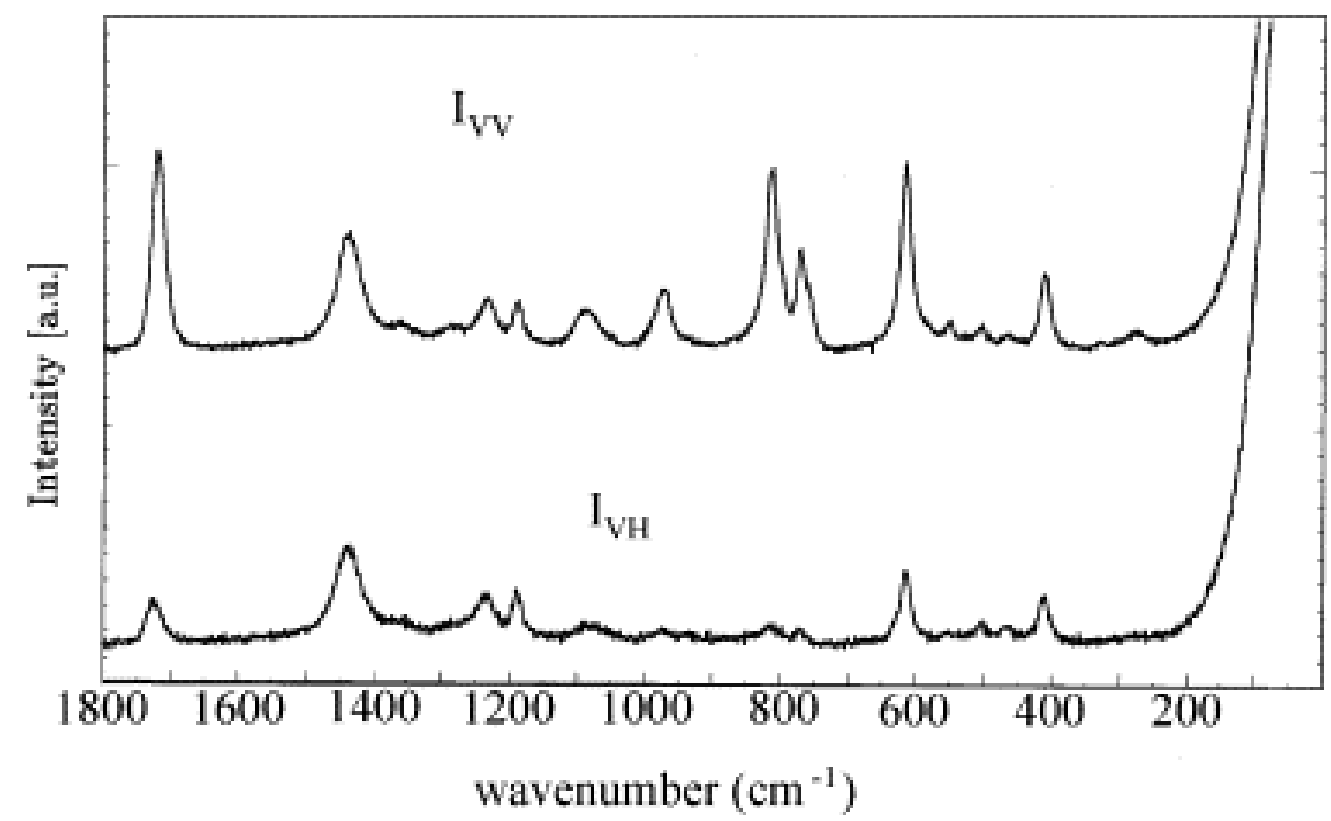

Fig 1 Polarized VV and VH Raman spectra of acetol in the $20-1800 \mathrm{~cm}^{-1}$ interval.

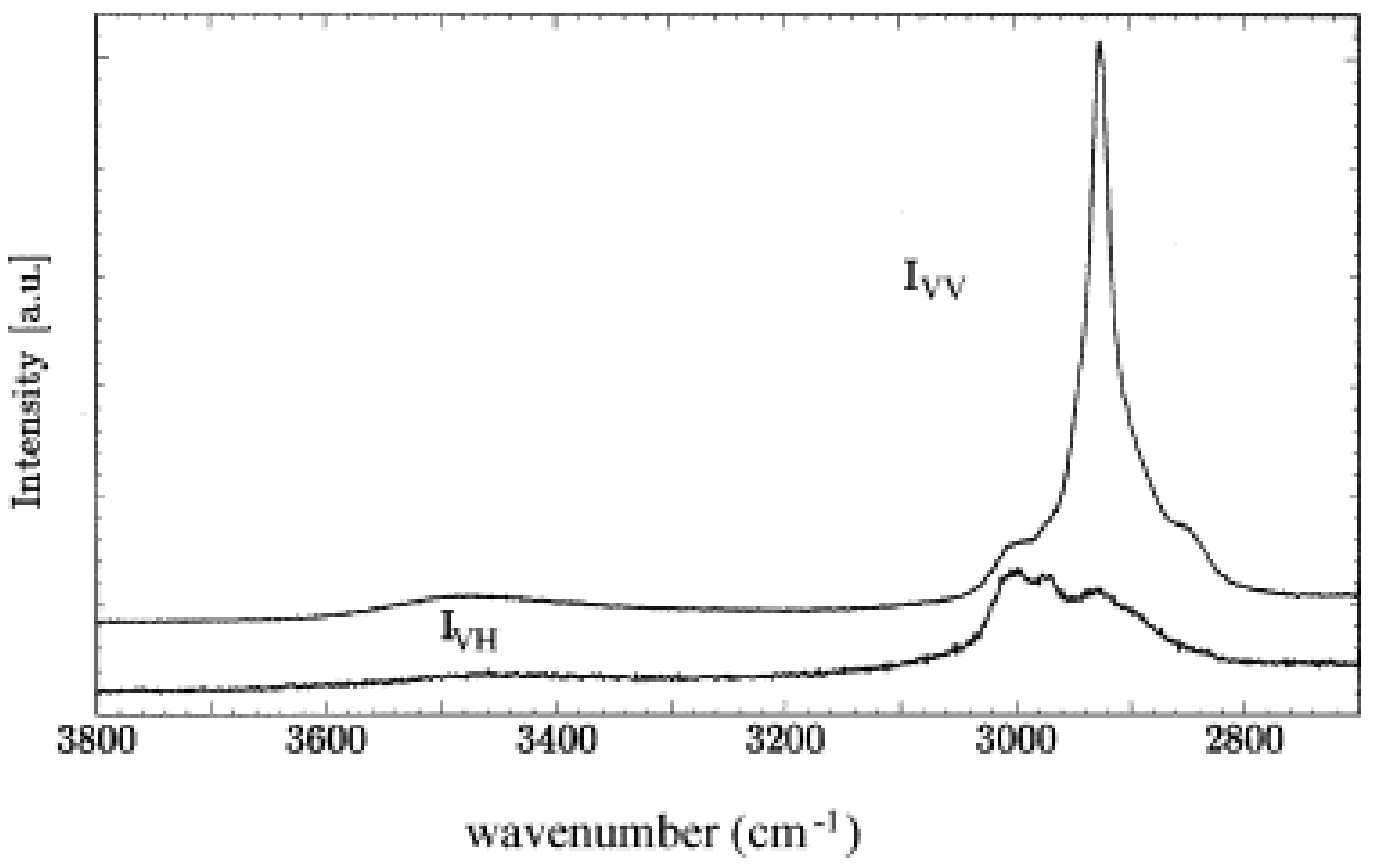

Fig 2 Polarized VV and VH Raman spectra of acetol in the $2700-3600 \mathrm{~cm}^{-1}$ interval. 
Mohaček-Grošev, V., (2005), "Vibrational analysis of hydroxyacetone" Spectrochimica Acta Part A: Molecular and Biomolecular Spectroscopy, Vol. 61, No. 3, pp. 477-484.

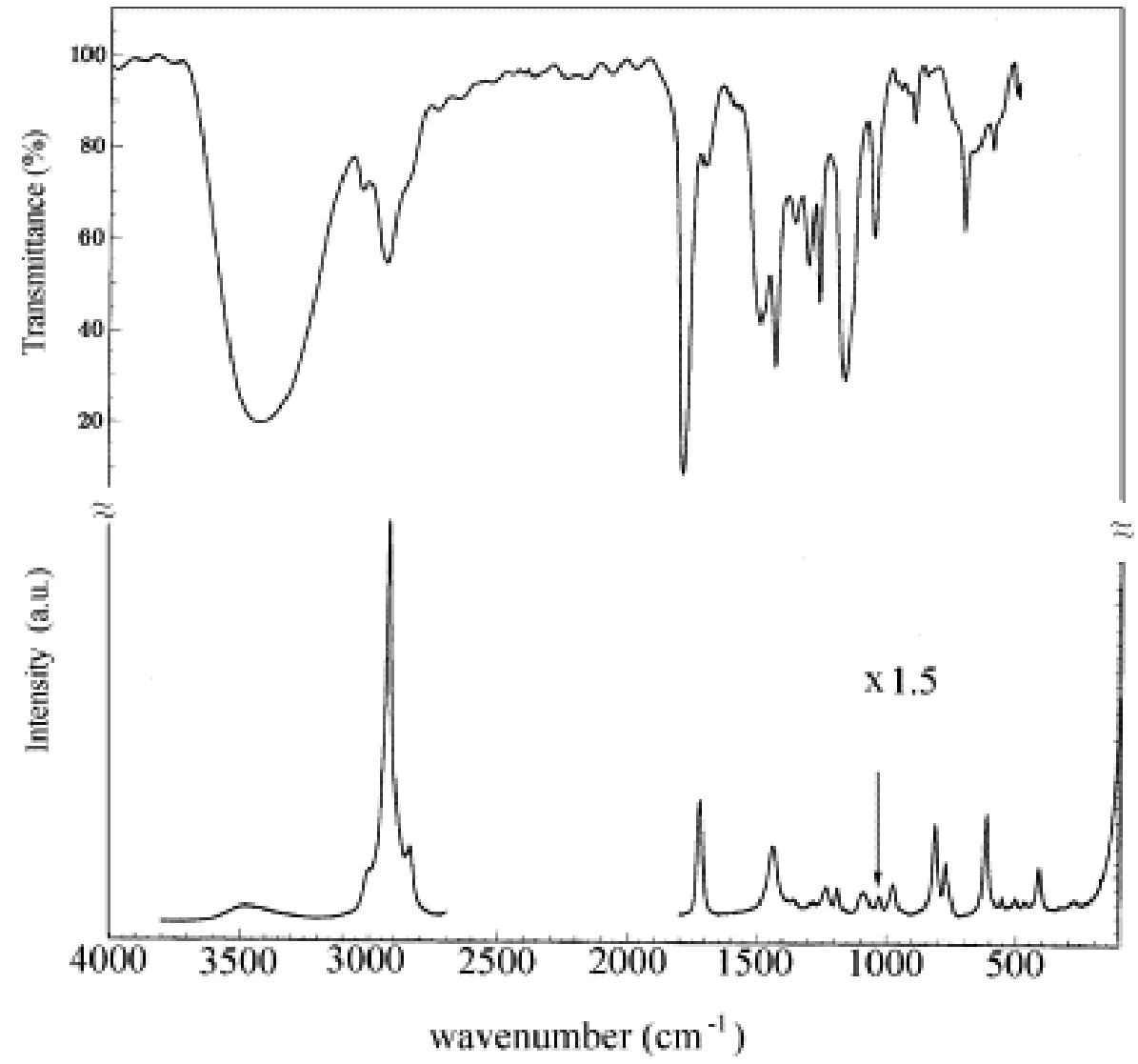

Fig 3 Infrared and Raman spectra of acetol previously warmed up in the stream of hot air for 15 min. Raman spectrum $\left(20-1800 \mathrm{~cm}^{-1}\right)$ is multiplied by factor 1.5 . The new Raman band at $1028.5 \mathrm{~cm}^{-1}$ is marked with an arrow.

Table 1 Observed and calculated vibrational frequencies of glycolaldehyde

\begin{tabular}{|c|c|c|c|c|}
\hline $\begin{array}{c}\text { Infrared } \\
\text { vapour [13] }\end{array}$ & $\begin{array}{c}\text { Infrared } \\
{[14]} \\
\text { Cc } \\
\text { conformer }\end{array}$ & $\begin{array}{c}\text { Ar matrix } \\
13 \text { K [14] } \\
\text { Tt conformer }\end{array}$ & $\begin{array}{c}\text { Calculated } \\
\text { glycolaldehye } \\
\text { frequencies } \\
\text { Cc conformer }\end{array}$ & Assignment \\
\hline & & $3668 \mathrm{~m}$ & & \\
\hline $3585 \mathrm{~m}$ & & & & \\
\hline $3565 \mathrm{~m}$ & $3549 \mathrm{w}$ & & 3605 & $\mathrm{O}-\mathrm{H}$ stretch. $\mathrm{A}^{\prime}$ \\
\hline $3546 \mathrm{~m}$ & $3541 \mathrm{~m}$ & & & \\
\hline & $3533 \mathrm{~m}$ & & & \\
\hline $2920 \mathrm{vw}$ & $2907 \mathrm{w}$ & $2940 \mathrm{w}$ & 2975 & $\mathrm{CH}_{2}$ asym. stretch. $\mathrm{A}^{\prime \prime}$ \\
\hline & & $2935 \mathrm{w}$ & & \\
\hline $2880 \mathrm{~m}$ & $2895 \mathrm{w}$ & & & \\
\hline $2835 \mathrm{~s}$ & $2845 \mathrm{~m}$ & & 2893 & $\mathrm{CH}_{2}$ sym. Stretch. $\mathrm{A}^{\prime}$ \\
\hline $2810 \mathrm{~s}$ & & $2810 \mathrm{vw}$ & 2808 & $\mathrm{C}-\mathrm{H}$ stretch. $\mathrm{A}^{\prime}$ \\
\hline
\end{tabular}


Mohaček-Grošev, V., (2005), "Vibrational analysis of hydroxyacetone" Spectrochimica Acta Part A: Molecular and Biomolecular Spectroscopy, Vol. 61, No. 3, pp. 477-484.

\begin{tabular}{|c|c|c|c|c|}
\hline \multirow[t]{2}{*}{$\begin{array}{c}\text { Infrared } \\
\text { vapour [13] }\end{array}$} & $\begin{array}{c}\text { Infrared } \\
{[14]} \\
\text { Cc } \\
\text { conformer }\end{array}$ & $\begin{array}{c}\text { Ar matrix } \\
13 \text { K [14] } \\
\text { Tt conformer }\end{array}$ & $\begin{array}{c}\begin{array}{c}\text { Calculated } \\
\text { glycolaldehye } \\
\text { frequencies }\end{array} \\
\text { Cc conformer }\end{array}$ & Assignment \\
\hline & $2712 \mathrm{w}$ & & & \\
\hline \multicolumn{5}{|l|}{1764 vs } \\
\hline 1753 vs & 1747 vs & 1747 vs & 1732 & C - O stretch. $\mathrm{A}^{\prime}$ \\
\hline \multicolumn{5}{|l|}{1742 vs } \\
\hline & $1700 \mathrm{~m}$ & & & \\
\hline & $1697 \mathrm{~m}$ & & & \\
\hline \multicolumn{5}{|l|}{$1468 \mathrm{~m}, \mathrm{br}$} \\
\hline $1440 \mathrm{~m}, \mathrm{br}$ & $1443 \mathrm{w}$ & $1439 \mathrm{~m}$ & 1422 & $\mathrm{CH}_{2}$ scissor. $\mathrm{A}^{\prime}$ \\
\hline \multirow[t]{3}{*}{$1410 \mathrm{~m}, \mathrm{br}$} & $1429 \mathrm{w}$ & & 1367 & $\mathrm{CH}_{2}$ def, $\mathrm{C}-\mathrm{O} \mathrm{A}^{\prime}$ \\
\hline & $1421 \mathrm{w}$ & & & \\
\hline & $1399 \mathrm{w}$ & & & \\
\hline $1376 \mathrm{~m}$ & $1367 \mathrm{~s}$ & & 1370 & $\mathrm{CH}_{2}$ def. $\mathrm{A}^{\prime \prime}$ \\
\hline $1359 \mathrm{w}$ & & $1353 \mathrm{w}$ & & \\
\hline \multicolumn{5}{|l|}{$1299 \mathrm{w}$} \\
\hline \multicolumn{5}{|l|}{$1282 \mathrm{R} \mathrm{m}$} \\
\hline \multicolumn{5}{|l|}{$1273 \mathrm{Q} \mathrm{m}$} \\
\hline \multicolumn{5}{|l|}{$1268 \mathrm{vw}$} \\
\hline $1266 \mathrm{~m}$ & $1267 \mathrm{vs}$ & & 1189 & $\mathrm{C}-\mathrm{O}-\mathrm{H}$ bend. $\mathrm{A}^{\prime}$ \\
\hline \multirow[t]{2}{*}{$1258 \mathrm{P} \mathrm{m}$} & $1231 \mathrm{vw}$ & & & \\
\hline & $1229 \mathrm{vw}$ & $1203 \mathrm{~m}, \mathrm{br}$ & & \\
\hline $1146 \mathrm{w}, \mathrm{sh}$ & $1130 \mathrm{w}$ & $1129 \mathrm{vw}$ & & \\
\hline $1117 \mathrm{R}, \mathrm{s}$ & $1110 \mathrm{vs}$ & & 1043 & C - O stretch. $\mathrm{A}^{\prime}$ \\
\hline \multicolumn{5}{|l|}{$1112 \mathrm{~s}$} \\
\hline & 1107 vs & & & \\
\hline \multicolumn{5}{|l|}{$1079 \mathrm{~m}$} \\
\hline $1059 \mathrm{~m}$ & & 1065 vs & 959 & $\mathrm{CH}_{2}$ def. $\mathrm{A}^{\prime}$ \\
\hline \multirow{2}{*}{$1050 \mathrm{~m}$} & & & 1025 & $\mathrm{CH}_{2}$ def. $\mathrm{A}^{\prime \prime}$ \\
\hline & & 998 vs & & \\
\hline \multicolumn{5}{|l|}{$916 \mathrm{w}$} \\
\hline \multicolumn{5}{|l|}{$871 \mathrm{~s}$} \\
\hline $861 \mathrm{~s}$ & $858 \mathrm{~s}$ & & 876 & $\mathrm{C}-\mathrm{C}$ stretch. $\mathrm{A}^{\prime}$ \\
\hline $859 \mathrm{~s}$ & $855 \mathrm{~s}$ & & & $\mathrm{C}-\mathrm{O}$ i.plane bend. $\mathrm{A}^{\prime}$ \\
\hline \multicolumn{5}{|l|}{$845 \mathrm{~s}$} \\
\hline $762 \mathrm{w}$ & & & 790 & C-H o.plane bend. A" \\
\hline $752 \mathrm{~m}$ & $749 \mathrm{~m}$ & & & \\
\hline
\end{tabular}


Mohaček-Grošev, V., (2005), "Vibrational analysis of hydroxyacetone" Spectrochimica Acta Part A: Molecular and Biomolecular Spectroscopy, Vol. 61, No. 3, pp. 477-484.

\begin{tabular}{|c|c|c|c|c|}
\hline $\begin{array}{c}\text { Infrared } \\
\text { vapour [13] }\end{array}$ & $\begin{array}{c}\text { Infrared } \\
{[14]} \\
\text { Cc } \\
\text { conformer }\end{array}$ & $\begin{array}{c}\text { Ar matrix } \\
13 \text { K [14] } \\
\text { Tt conformer }\end{array}$ & $\begin{array}{c}\text { Calculated } \\
\text { glycolaldehye } \\
\text { frequencies }\end{array}$ & Assignment \\
\hline $750 \mathrm{w}$ & & & & \\
\hline $748 w$ & & & & \\
\hline $746 w$ & & & & \\
\hline $743 \mathrm{w}$ & & & & \\
\hline $568 \mathrm{vw}$ & & & & \\
\hline $550 \mathrm{vw}$ & & $538 \mathrm{w}$ & 459 & $\mathrm{O}-\mathrm{C}-\mathrm{C}$ bend. $\mathrm{A}^{\prime}$ \\
\hline $535 \mathrm{vw}$ & & $535 \mathrm{w}$ & & \\
\hline & $341 \mathrm{vvs}$ & $331 \mathrm{~m}$ & 396 & $\mathrm{OH}$ torsion $\mathrm{A}^{\prime \prime}$ \\
\hline $260^{\mathrm{a}}$ & $274 \mathrm{~m}$ & & 188 & $\begin{array}{l}\mathrm{C}-\mathrm{H} \text { i.p. bend }+\mathrm{C}-\mathrm{O} \\
\text { i.p. bend } \mathrm{A}^{\prime}\end{array}$ \\
\hline $195^{\mathrm{a}}$ & & & 224 & $\begin{array}{l}\mathrm{CH}_{2} \mathrm{OH} \text { torsion around } \\
\mathrm{C}-\mathrm{C} \text { bond, } \mathrm{A}^{\prime \prime}\end{array}$ \\
\hline
\end{tabular}

a From microwave study of Marstokk and Møllendal [22].

Table 2 Observed and calculated vibrational frequencies of hydroxyacetone

\begin{tabular}{|c|c|c|c|}
\hline $\begin{array}{l}\text { Infrared liquid } \\
\text { acetol }\end{array}$ & $\begin{array}{l}\text { Raman liquid } \\
\text { acetol }\end{array}$ & $\begin{array}{l}\text { Calculated hydroxyacetone } \\
\text { frequencies }\end{array}$ & Assignment \\
\hline $3405 \mathrm{vs,vbr}$ & $3470 \mathrm{w}, \mathrm{vbr}$ & 3605 & O-H stretch. A' \\
\hline \multirow[t]{4}{*}{$2997 \mathrm{~m}$} & 2996 w,br & 3020 & $\mathrm{CH}_{3}$ asym. stretch. $\mathrm{A}^{\prime}$ \\
\hline & $2974 \mathrm{w}, \mathrm{sh}$ & 3018 & $\mathrm{CH}_{3}$ asym. stretch. $\mathrm{A}^{\prime \prime}$ \\
\hline & & 2974 & $\mathrm{CH}_{2}$ asym. stretch. $\mathrm{A}^{\prime \prime}$ \\
\hline & 2926 vs & 2966 & $\mathrm{CH}_{3}$ sym. stretch. $\mathrm{A}^{\prime}$ \\
\hline \multirow[t]{2}{*}{2900 s,br } & $2900 \mathrm{w}, \mathrm{sh}$ & 2901 & $\mathrm{CH}_{2}$ sym. stretch. $\mathrm{A}^{\prime}$ \\
\hline & $2854 \mathrm{mw}$ & & Overtone $\mathrm{CH}_{3}$ bending \\
\hline \multicolumn{4}{|l|}{$2809 \mathrm{~m}, \mathrm{sh}$} \\
\hline 1724 vs & $1720 \mathrm{~s}$ & 1751 & $\mathrm{C}-\mathrm{O}$ stretching $\mathrm{A}^{\prime}$ \\
\hline $1641 \mathrm{~m}$ & & & Water OHO bending \\
\hline \multicolumn{4}{|l|}{$1534 \mathrm{w}$} \\
\hline & $1435 \mathrm{~ms}, \mathrm{br}$ & 1459 & $\mathrm{CH}_{2}$ scissoring $\mathrm{A}^{\prime}$ \\
\hline \multirow[t]{2}{*}{$1422 \mathrm{~s}, \mathrm{br}$} & & 1400 & $\mathrm{HCH}$ bend. in $\mathrm{CH}_{3} \mathrm{~A}^{\prime \prime}$ \\
\hline & & 1362 & $\mathrm{CH}_{2}$ def. $\mathrm{A}^{\prime \prime}$ \\
\hline $1361 \mathrm{~s}$ & $1361 \mathrm{w}$ & 1402 & Sym. $\mathrm{CH}_{3}$ bend. $\mathrm{A}^{\prime}$ \\
\hline \multicolumn{4}{|l|}{$1287 \mathrm{~m}$} \\
\hline & $1283 \mathrm{w}, \mathrm{br}$ & 1394 & $\begin{array}{l}\mathrm{HCH} \text { bend in } \mathrm{CH}_{2} \text { and } \\
\mathrm{CH}_{3} \mathrm{~A}^{\prime}\end{array}$ \\
\hline
\end{tabular}


Mohaček-Grošev, V., (2005), "Vibrational analysis of hydroxyacetone" Spectrochimica Acta Part A: Molecular and Biomolecular Spectroscopy, Vol. 61, No. 3, pp. 477-484.

\begin{tabular}{|c|c|c|c|}
\hline $\begin{array}{l}\text { Infrared liquid } \\
\text { acetol }\end{array}$ & $\begin{array}{c}\text { Raman liquid } \\
\text { acetol }\end{array}$ & $\begin{array}{l}\text { Calculated hydroxyacetone } \\
\text { frequencies }\end{array}$ & Assignment \\
\hline $1233 \mathrm{~m}$ & $1233.5 \mathrm{~m}, \mathrm{br}$ & 1207 & $\mathrm{COH}$ bend. $\mathrm{A}^{\prime}$ \\
\hline $1189 \mathrm{~ms}$ & $1189.5 \mathrm{~m}$ & 1248 & CC stretch. $\mathrm{A}^{\prime}$ \\
\hline $1083 \mathrm{~s}, \mathrm{br}$ & $1086.5 \mathrm{~m}, \mathrm{br}$ & 1093 & $\mathrm{CO}+\mathrm{COH} \mathrm{A}$ \\
\hline \multirow[t]{2}{*}{$1057 \mathrm{~s}, \mathrm{sh}$} & $1053 \mathrm{mw}, \mathrm{sh}$ & & \\
\hline & & 988 & $\mathrm{CH}_{2}$ def. $\mathrm{A}^{\prime \prime}$ \\
\hline $973 \mathrm{~m}$ & $975.5 \mathrm{~m}, \mathrm{br}$ & 982 & $\mathrm{CH}_{2}$ def. $+\mathrm{CO} \mathrm{A}^{\prime}$ \\
\hline \multicolumn{4}{|l|}{$888 \mathrm{vw}$} \\
\hline $870 \mathrm{vw}$ & $872 \mathrm{vw}$ & 873 & $\mathrm{CH}_{3}$ rocking $\mathrm{A}^{\prime \prime}$ \\
\hline \multicolumn{4}{|l|}{$841 \mathrm{w}$} \\
\hline $816 \mathrm{~mW}$ & $813 \mathrm{~s}$ & 864 & $\mathrm{CC}$ str. $+\mathrm{CH}_{3}$ bend. $\mathrm{A}^{\prime}$ \\
\hline $768 \mathrm{vw}$ & $771 \mathrm{~ms}$ & 744 & $\mathrm{CH}_{3}+\mathrm{CC}+\mathrm{CH}_{3}$ def. $\mathrm{A}^{\prime}$ \\
\hline $750 \mathrm{vw}$ & $758 \mathrm{~m}, \mathrm{sh}$ & & \\
\hline $615 \mathrm{~m}$ & $617 \mathrm{~s}$ & 465 & $\begin{array}{l}\text { C-O i.p. bend + OCC } \\
\text { bend } A^{\prime}\end{array}$ \\
\hline \multirow[t]{2}{*}{$580 \mathrm{mw}, \mathrm{vbr}$} & & & Water libration \\
\hline & $552 \mathrm{w}$ & & \\
\hline \multirow[t]{2}{*}{$503 \mathrm{mw}$} & $504 \mathrm{w}$ & 440 & $\mathrm{C}-\mathrm{O}$ o.p. bend $+\tau_{\mathrm{OH}} \mathrm{A}^{\prime \prime}$ \\
\hline & $465 \mathrm{w}$ & & \\
\hline \multirow[t]{5}{*}{$418 \mathrm{mw}$} & $411 \mathrm{~m}$ & 395 & CCC bend $\mathrm{A}^{\prime}$ \\
\hline & $330 \mathrm{w}$ & 362 & $\tau_{\mathrm{OH}}+\mathrm{C}-\mathrm{O}$ o.p. bend. A" \\
\hline & $276 \mathrm{w}, \mathrm{br}$ & 242 & $\begin{array}{l}\text { OCC bend. + C - O i.p. } \\
\text { bend } \mathrm{A}^{\prime}\end{array}$ \\
\hline & & 177 & $\tau_{\mathrm{CH} 2 \mathrm{OH}}{ }^{\mathrm{a}} \mathrm{A}^{\prime \prime}$ \\
\hline & $80^{\underline{b}}$ w,br & 65 & $\tau_{\mathrm{CH} 3}{ }^{\underline{a}} \mathrm{~A}^{\prime \prime}$ \\
\hline
\end{tabular}

a These refer to results of normal mode calculation for free molecule, the band at $80 \mathrm{~cm}^{-1}$ is assigned to $\mathrm{CH}_{2} \mathrm{OH}$ torsion in hydroxyacetone solution.

b Observed in temperature reduced Raman spectrum.

Geometrical parameters needed for normal coordinate calculations were taken from microwave study of glycolaldehyde by Marstokk and Møllendal [21] and [22] and for hydroxyacetone from the microwave study by Kattija-Ari and Harmony [23]. Both molecules have the plane of symmetry in the gas phase and the calculated frequencies refer to these Cc conformers. The internal coordinates of the two molecules are depicted in Fig. 4 and Fig. 5. Force constants used in the calculation are listed in Table 3 and Table 4. 


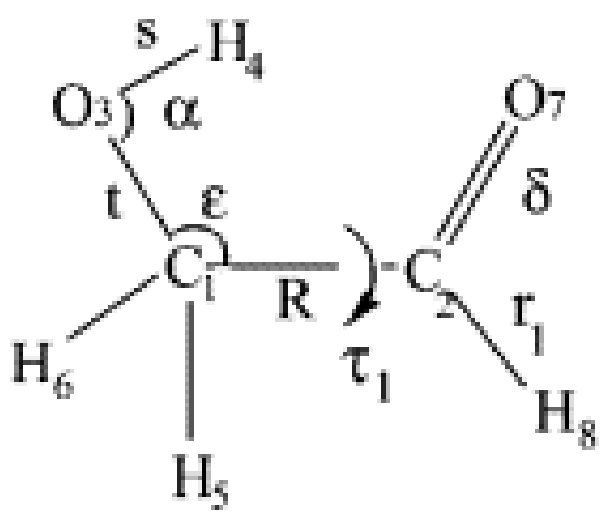

$\beta_{1}+\mathrm{H}_{5} \mathrm{C}_{1} \mathrm{C}_{2}$

$\mathrm{B}_{3} * \mathrm{H}_{5} \mathrm{C}_{1} \mathrm{O}_{3}$

$\delta \mathrm{C}=\mathrm{O}$ i.p. bend.

$\phi \quad \mathrm{C}_{2}-\mathrm{H}_{8}$ i.p. bend.

$\lambda \mathrm{C}_{2}-\mathrm{H}_{8}$ o.p. bend.

$\tau_{1} \mathrm{CH}_{2} \mathrm{O}$ torsion

$\tau_{2} \mathrm{O}_{3} \mathrm{H}_{4}$ torsion

Fig 4 Definition of internal coordinates for glycolaldehyde molecule.

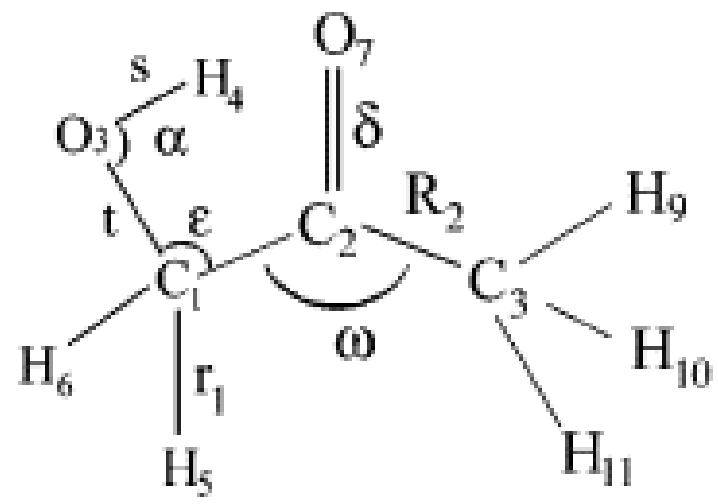

$\beta_{1} \Varangle \mathrm{H}_{5} \mathrm{C}_{1} \mathrm{C}_{2}$

$\mathrm{B}_{3} \star \mathrm{H}_{5} \mathrm{C}_{1} \mathrm{O}_{3}$

$\delta \quad \mathrm{C}=\mathrm{O}$ i.p. bend.

$\theta_{9} \Varangle \mathrm{H}_{9} \mathrm{C}_{3} \mathrm{C}_{2}$

$\alpha_{9} \star \mathrm{H}_{10} \mathrm{C}_{3} \mathrm{H}_{11}$

$\pi \mathrm{C}=\mathrm{O}$ o.p. bend.

$\tau_{1} \mathrm{CH}_{2} \mathrm{O}$ torsion

$\tau_{2} \mathrm{OH}$ torsion

$\tau_{3} \mathrm{CH}_{3}$ torsion

Fig 5 Definition of internal coordinates for hydroxyacetone.

Table 3 Diagonal force constants used for glycolaldehyde (GHD) and hydroxyacetone (HA) ( $K$ in $\mathrm{N} \mathrm{cm}^{-1}, H_{\text {in }} 10^{-16}$ $\mathrm{N} \mathrm{cm} \mathrm{rad}^{-2}$ )

\begin{tabular}{|l|l|l|l|}
\hline $\begin{array}{c}\text { Force } \\
\text { constant }\end{array}$ & Description & $\begin{array}{c}\text { Value used } \\
\text { (GHD and } \\
\text { HA) }\end{array}$ & $\begin{array}{c}\text { Value assigned in parent molecule-if } \\
\text { different (parent molecule, reference) }\end{array}$ \\
\hline$K_{\mathrm{S}}$ & $\mathrm{OH}$ stretching & 7.2 & $7.337(\mathrm{EG}=$ ethylene glycol, [25]) \\
\hline$K_{r 2}$ & $\mathrm{CH}_{2}$ stretching & 4.654 & (EG) \\
\hline
\end{tabular}


Mohaček-Grošev, V., (2005), "Vibrational analysis of hydroxyacetone" Spectrochimica Acta Part A: Molecular and Biomolecular Spectroscopy, Vol. 61, No. 3, pp. 477-484.

\begin{tabular}{|c|c|c|c|}
\hline $\begin{array}{l}\text { Force } \\
\text { constant }\end{array}$ & Description & $\begin{array}{c}\text { Value used } \\
\text { (GHD and } \\
\text { HA) }\end{array}$ & $\begin{array}{l}\text { Value assigned in parent molecule-if } \\
\text { different (parent molecule, reference) }\end{array}$ \\
\hline$K_{r 9}$ & $\mathrm{CH}_{3}$ stretching & 4.882 & (acetone AN, [24]) \\
\hline$K_{\mathrm{H} 8}$ & $\mathrm{C}_{2}-\mathrm{H}_{8}$ str. glyc. & 4.243 & 4.273(acetaldehyde AD, [24]) \\
\hline$K_{\mathrm{d}}$ & C $-\mathrm{O}$ stretching & 10.77 and 9.9 & (AN) \\
\hline$K_{\mathrm{t}}$ & $\mathrm{C}-\mathrm{O}$ stretching & 5.044 & (EG) \\
\hline$K_{\mathrm{R}}$ & C $-\mathrm{C}$ stretching & 4.760 & (EG) \\
\hline$H_{\omega}$ & $\begin{array}{l}\mathrm{C}-\mathrm{C}-\mathrm{C} \\
\text { bending }\end{array}$ & 1.647 & (AN) \\
\hline$H_{\alpha}$ & $\begin{array}{l}\mathrm{C}-\mathrm{O}-\mathrm{H} \\
\text { bending }\end{array}$ & 0.8 & 0.762 (EG) \\
\hline$H_{\varepsilon}$ & $\begin{array}{l}\mathrm{O}-\mathrm{C}-\mathrm{C} \\
\text { bending }\end{array}$ & 1.060 & (EG) \\
\hline$H_{\beta}$ & $\mathrm{CH}_{2}$ bending & 0.65 & 0.71 (EG) \\
\hline$H_{\gamma}$ & $\begin{array}{l}\mathrm{HCH} \text { bend in } \\
\mathrm{CH}_{2}\end{array}$ & 0.50 & 0.578 (EG) \\
\hline$H_{\theta}$ & $\begin{array}{l}\mathrm{H}-\mathrm{C}-\mathrm{C} \text { bend } \\
\text { in } \mathrm{CH}_{3}\end{array}$ & 0.45 & $0.468(\mathrm{AN})$ \\
\hline$H_{\alpha}$ & $\begin{array}{l}\mathrm{HCH} \text { bend in } \\
\mathrm{CH}_{3}\end{array}$ & 0.49 & $0.521(\mathrm{AN})$ \\
\hline$H_{\delta}$ & $\begin{array}{l}\mathrm{O}-\mathrm{CC} \text { in plane } \\
\text { bend. }\end{array}$ & 1.006 & (EG) \\
\hline$H_{\tau 1}$ & $\mathrm{OH}$ torsion & 0.009 & 0.049 (EG) \\
\hline$H_{\tau 2}$ & $\mathrm{CH}_{2} \mathrm{OH}$ torsion & 0.004 & 0.123 (EG) \\
\hline$H_{\tau 3}$ & $\mathrm{CH}_{3}$ torsion & 0.007 & $(\mathrm{AN})$ \\
\hline$H_{\pi}$ & $\begin{array}{l}\mathrm{C}-\mathrm{O} \text { out of } \\
\text { plane bend. }\end{array}$ & 0.217 & (AN) \\
\hline$H_{\varphi}$ & $\begin{array}{l}\mathrm{C}_{2}-\mathrm{H}_{8} \text { in } \mathrm{pl} \\
\text { bend. }\end{array}$ & 0.435 & (AD) \\
\hline$H_{\lambda}$ & $\begin{array}{l}\mathrm{C}_{2}-\mathrm{H}_{8} \text { out of } \\
\text { pl. bend. }\end{array}$ & 0.296 & $(\mathrm{AD})$ \\
\hline
\end{tabular}

Table 4 Interaction force constants used for glycolaldehyde and hydroxyacetone $\left(f_{r r}\right.$ in $\mathrm{N} \mathrm{cm}^{-1} f_{\beta, \beta}$ in $10^{-16} \mathrm{~N} \mathrm{~cm}^{-1}$ $\operatorname{rad}^{-2}$, and $f_{\mathrm{R} \theta}, f_{\mathrm{t} \beta}$ in $10^{-8} \mathrm{~N} \mathrm{rad}^{-1}$ )

\begin{tabular}{|l|l|l|l|}
\hline \multicolumn{1}{|c|}{$\begin{array}{c}\text { Force } \\
\text { constant }\end{array}$} & \multicolumn{1}{|c|}{ Description } & $\begin{array}{c}\text { Value } \\
\text { used }\end{array}$ & \multicolumn{1}{|c|}{$\begin{array}{c}\text { Value assigned in parent molecule-if } \\
\text { different (parent molecule, reference) }\end{array}$} \\
\hline$f_{r 2, r 3}$ & $\begin{array}{l}\mathrm{CH}_{2} \text { stretch. } \\
\text { inter. }\end{array}$ & -0.013 & Ethylene glycol (EG, [25]) \\
\hline$f_{r 9, r 10}$ & $\begin{array}{l}\mathrm{CH}_{3} \text { stretch. } \\
\text { inter. }\end{array}$ & 0.073 & Acetone (AN, [24]) \\
\hline$f_{\mathrm{t}, \mathrm{R}}$ & $\mathrm{CO} / \mathrm{CC}$ stretch. & 0.248 & $(\mathrm{EG})$ \\
\hline
\end{tabular}


Mohaček-Grošev, V., (2005), "Vibrational analysis of hydroxyacetone" Spectrochimica Acta Part A: Molecular and Biomolecular Spectroscopy, Vol. 61, No. 3, pp. 477-484.

\begin{tabular}{|l|l|l|l|}
\hline $\begin{array}{c}\text { Force } \\
\text { constant }\end{array}$ & \multicolumn{1}{|c|}{ Description } & \multicolumn{1}{|c|}{$\begin{array}{c}\text { Value } \\
\text { used }\end{array}$} & \multicolumn{1}{|c|}{$\begin{array}{c}\text { Value assigned in parent molecule-if } \\
\text { different (parent molecule, reference) }\end{array}$} \\
\hline$f_{\mathrm{t} \beta 3}, f_{\mathrm{t} \beta 4}$ & $\mathrm{CO} / \beta_{3}, \mathrm{CO} / \beta_{4}$ & 0.076 & $(\mathrm{EG})$ \\
\hline$f_{\mathrm{R}, \theta}$ & $\begin{array}{l}\mathrm{CC} \text { stretch., } \mathrm{CH} \mathrm{CH}_{3} \\
\text { bend. }\end{array}$ & 0.3 & \\
\hline$f_{\beta 1 \beta 2}$ & $\beta_{1} / \beta_{2}$ & -0.168 & $0.068(\mathrm{EG})$ \\
\hline$f_{\beta 1 \beta 3}$ & $\beta_{1} / \beta_{3}$ & 0.02 & $-0.02(\mathrm{EG})$ \\
\hline$f_{\beta 1 \beta 4}$ & $\beta_{1} / \beta_{4}$ & 0.023 & $(\mathrm{EG})$ \\
\hline$f_{\beta \varphi}$ & $\beta_{1} / \varphi$ & 0.1 & \\
\hline$f_{\beta \theta 10}$ & $\beta_{2} / \theta_{10}$ & 0.1 & \\
\hline$f_{\theta 9, \theta 10}$ & $\theta_{9} / \theta_{10}$ & -0.021 & $(\mathrm{AN})$ \\
\hline
\end{tabular}

\subsection{Glycoladehyde}

At present there exist differences in the assignment of glycolaldehyde bands-Michelsen and Klaboe [13] based their tentative assignment on the infrared spectrum of vapor, while Aspiala et al. [14] studied glycolaldehyde in $\mathrm{Ar}, \mathrm{Kr}$ and Xe matrices by infrared spectroscopy. The greatest difference in assignment concerns $\mathrm{O} \square \mathrm{CC}$ in plane bending mode. In the vapour it was assigned to $859 \mathrm{~cm}^{-1}$ infrared band [13], and in Ar matrix to $749 \mathrm{~cm}^{-1}$ band for $\mathbf{C c}$ conformer [14]. The band at $535 \mathrm{~cm}^{-1}$ in Ar matrix was attributed to $\mathrm{O} \square \mathrm{CC}$ bending mode of $\mathbf{T t}$ conformer [14]. Michelsen and Klaboe assigned the $1266 \mathrm{~cm}^{-1}$ band to $\mathrm{C} \square \mathrm{O}$ stretching mode $(v(\mathrm{CO}))$ and the $1059 \mathrm{~cm}^{-1}$ band to $\mathrm{C} \square \mathrm{O} \square \mathrm{H}$ bending $(\delta(\mathrm{COH}))$ [13], while Aspiala et al. attribute 1267 and $1110 \mathrm{~cm}^{-1}$ bands in argon matrix to $\mathrm{C} \square \mathrm{O} \square \mathrm{H}$ bending and $\mathrm{C} \square \mathrm{O}$ stretching of $\mathbf{C c}$ conformer [14]. For the other conformer present in the matrix, Tt conformer, $\delta(\mathrm{COH})$ is assigned to $1203 \mathrm{~cm}^{-1}$ and $v(\mathrm{CO})$ to $1065 \mathrm{~cm}^{-1}$ band [14]. The lowest frequency band observed in vapour was a weak band at $550 \mathrm{~cm}^{-1}$, attributed to $\delta(\mathrm{CCO})$ bending of $\mathbf{C c}$ conformer. In argon matrix a very strong band at $341 \mathrm{~cm}^{-1}$ was identified as $\mathrm{OH}$ torsion, and a medium band at $274 \mathrm{~cm}^{-1}$ as $\delta(\mathrm{CCO})$ of $\mathbf{C c}$ conformer. For Tt conformer in Ar matrix, a weak band at $535 \mathrm{~cm}^{-1}$ was taken to correspond to $\delta(\mathrm{O} \square \mathrm{CC})$ bending mode, while $\delta(\mathrm{CCO})$ bending mode was attributed to a medium band at $331 \mathrm{~cm}^{-1}$.

Altogether there are $12 \mathrm{~A}^{\prime}$ and $6 \mathrm{~A}^{\prime \prime}$ modes belonging to $\mathrm{C}_{\mathrm{s}}$ group that are calculated (see Table 1). The $\mathrm{O} \square \mathrm{CC}$ in plane bending mode is calculated at $188 \mathrm{~cm}^{-1}$, which differs from the previously mentioned assignments, but is more in accord with the result from microwave study which reported the existence of a low in plane bending mode at $260 \mathrm{~cm}^{-1}$ and a torsional mode at $195 \mathrm{~cm}^{-1}$ [22]. The $\delta(\mathrm{COH})$ mode was assigned to $1266 \mathrm{~cm}^{-1}$ vapour band, and the $\delta(\mathrm{CCO})$ mode to the $550 \mathrm{~cm}^{-1}$ band. The strong vapour band at $861 \mathrm{~cm}^{-1}$ is dominantly $\mathrm{C} \square \mathrm{C}$ stretching mode. Torsional transitions were calculated at $396 \mathrm{~cm}^{-1}(\tau \mathrm{OH})$ and $224 \mathrm{~cm}^{-1}\left(\tau \mathrm{CH}_{2} \mathrm{O}\right)$. 


\subsection{Hydroxyacetone}

Hydroxyacetone is extremely hygroscopic substance. In a few days there is so much water absorbed from the air, that the intensity ratio of 1740 and $1643 \mathrm{~cm}^{-1}$ infrared bands changes from 10 to 2 suggesting the increase of water content from 10 to 50\%. Also, there is a high fluorescence emanating from the substance when illuminated with $514.5 \mathrm{~nm}$ laser light. One needs to wait for several hours before the system relaxed into the ground state and the level of background signal dropped enough for Raman spectra to be acquired. At low temperatures the fluorescent background signal could not be eliminated.

The results of normal mode calculation for $17 \mathrm{~A}^{\prime}$ and $10 \mathrm{~A}^{\prime \prime}$ vibrations of the $\mathbf{C c}$ conformer are given in Table 2. For the majority or high frequency bands, the calculated values agree more or less with those of the corresponding glycolaldehyde conformer. The bands corresponding to $\mathrm{COH}$ bending and $\mathrm{C} \square \mathrm{O}$ stretching mode lie at $30 \mathrm{~cm}^{-1}$ lower wavenumbers than in glycolaldehyde, but the calculated value for $\mathrm{C} \square \mathrm{O}$ stretching is higher for glycolaldehyde than for hydroxyacetone. There are two bands both in infrared $\left(1083\right.$ and $\left.1057 \mathrm{~cm}^{-1}\right)$ and in Raman spectrum (1086.5 and $1053 \mathrm{~cm}^{-1}$ ) that are assigned to $v(\mathrm{C} \square \mathrm{O})$, and this is one of the indicators of several hydroxyacetone conformers in the solution.

The broad water libration band observed at $\sim 500 \mathrm{~cm}^{-1}$ in the infrared spectrum overlaps with the lower frequency acetol bands: $\mathrm{C} \square \mathrm{O}$ in plane bending, $\mathrm{CCC}$ bending, $\mathrm{C} \square \mathrm{O}$ out of plane bending, OCC bending and hydroxyl torsion. The in plane $\mathrm{C} \square \mathrm{O}$ bending mode is observed at $617 \mathrm{~cm}^{-1}$ in Raman and at $615 \mathrm{~cm}^{-1}$ in the infrared spectrum of liquid acetol, while the $\mathrm{C} \square \mathrm{O}$ out of plane bending mode is attributed to $503 \mathrm{~cm}^{-1}$ infrared and $504 \mathrm{~cm}^{-1}$ Raman band. The CCC bending mode is assigned to $418 \mathrm{~cm}^{-1}$ infrared and $411 \mathrm{~cm}^{-1}$ Raman band (in acetone it is $483 \mathrm{~cm}^{-1}$ ). Torsion of the hydroxyl group is calculated at $362 \mathrm{~cm}^{-1}$ and taken to correspond to a weak Raman band centered at $330 \mathrm{~cm}^{-1}$. The OCC in plane bending mode is calculated to be $242 \mathrm{~cm}^{-1}$ and assigned to broad and weak $270 \mathrm{~cm}^{-1}$ Raman band.

Of the two lowest internal modes, $\mathrm{CH}_{2} \mathrm{OH}$ torsion and $\mathrm{CH}_{3}$ torsion, only one seems to be observed at $80 \mathrm{~cm}^{-1}$ in Raman spectra of acetol (see Fig. 6 and Fig. 7). The low frequency Raman spectrum of liquid was divided by temperature dependent factor contributing to Raman scattered intensity [30]:

$$
\left(\omega_{0}-\omega_{i}\right)^{4} \omega_{i}^{-1}\left(1-\exp \left(\frac{h \omega_{i} c}{k T}\right)\right), B_{\mathrm{CH}_{3}}
$$

where $\omega_{0}$ is the absolute frequency (in wavenumbers), $\omega_{i}$ the Raman shift, $h$ the Planck's constant, and $k$ the Boltzmann's constant. The resulting intensity function is usually denoted $R(\omega)$. Methyl libration bands are notoriously weak in Raman spectra [34], therefore the $80 \mathrm{~cm}^{-1}$ band is attributed to hydroxymethyl torsions. 
Mohaček-Grošev, V., (2005), "Vibrational analysis of hydroxyacetone" Spectrochimica Acta Part A: Molecular and Biomolecular Spectroscopy, Vol. 61, No. 3, pp. 477-484.

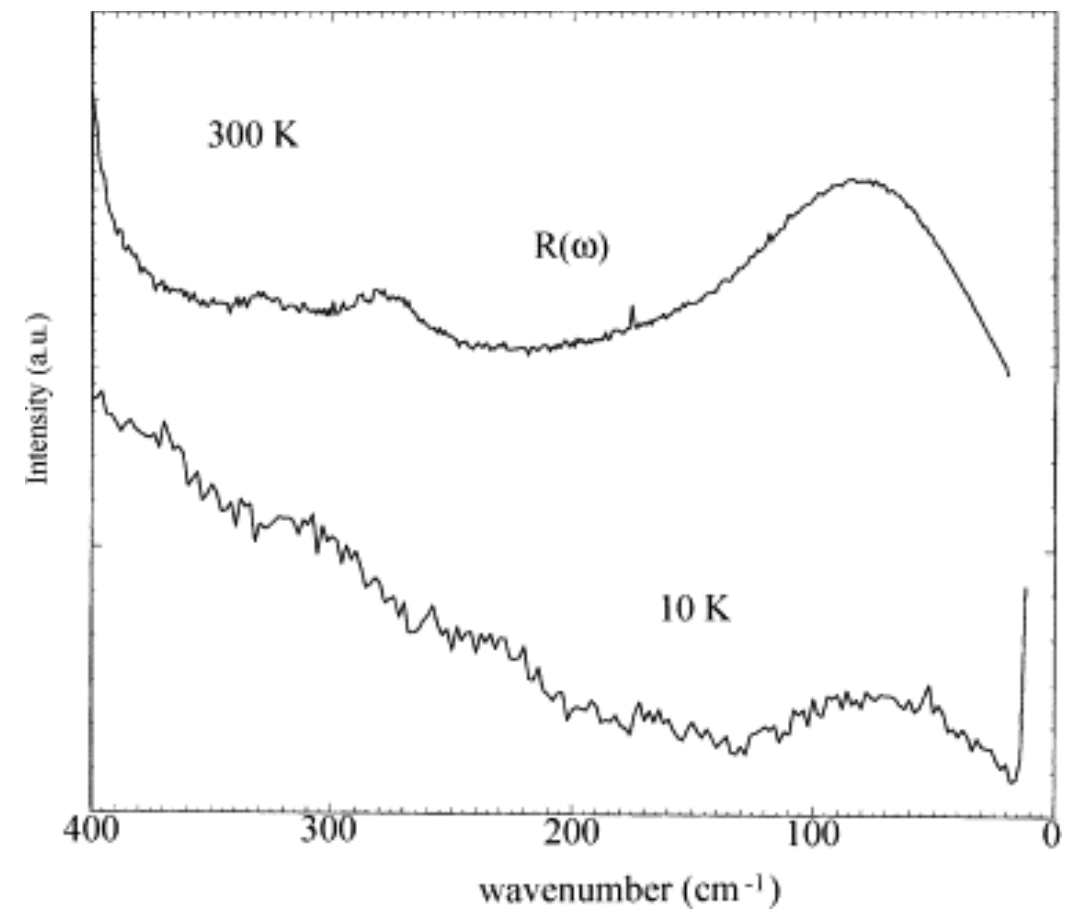

Fig 7 Temperature reduced Raman spectrum $R(\omega)$ of acetol at room temperature (300 K) (see text for an explanation), and low frequency Raman spectrum for a solid at $10 \mathrm{~K}$.

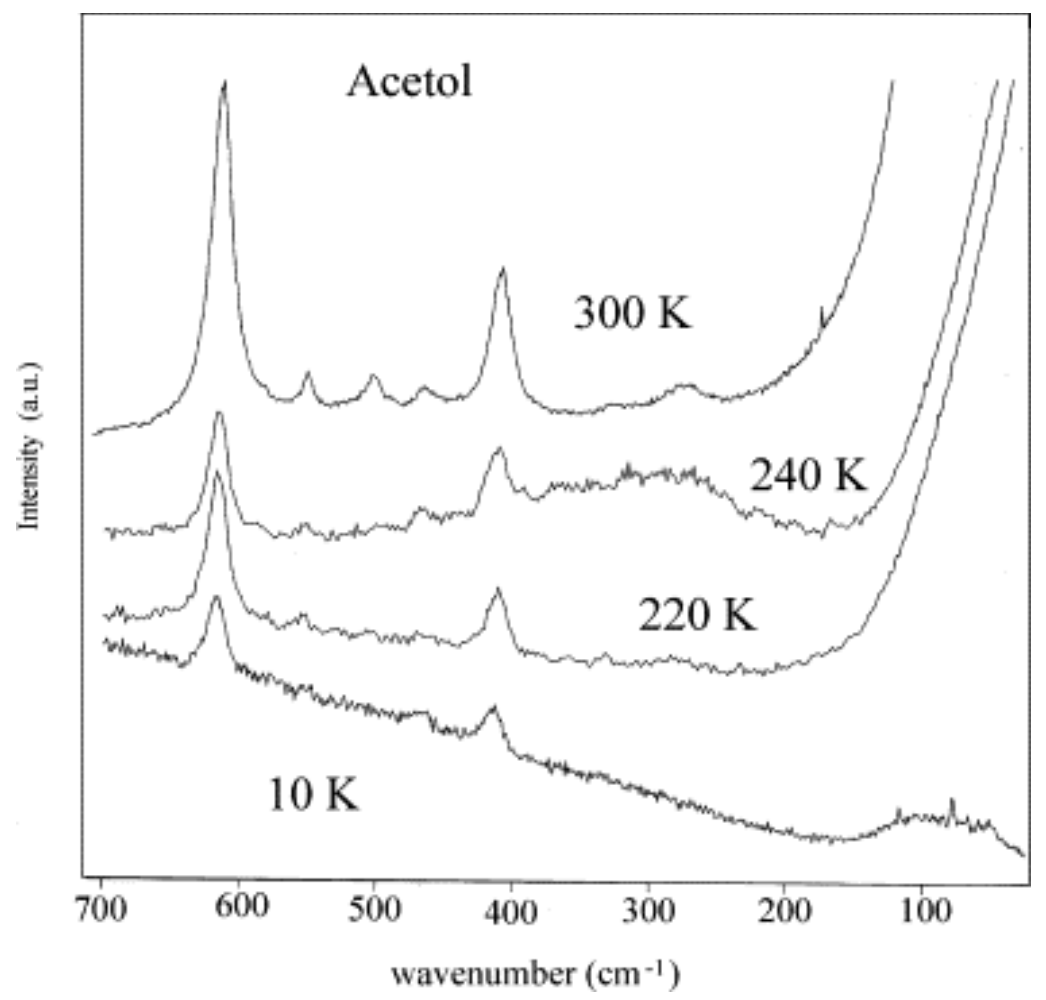

Fig 1 Temperature dependence of low frequency Raman spectra of acetol $\left(20-700 \mathrm{~cm}^{-1}\right)$.

There are still two unassigned Raman bands of acetol: one at $465 \mathrm{~cm}^{-1}$ and the other at $552 \mathrm{~cm}^{-1}$. 


\section{Discussion}

It was pointed out [23] that the barrier to methyl group rotation in hydroxyacetone is significantly lower (it is $810 \mathrm{~J} \mathrm{~mol}^{-1}$ ) than in other similar compounds such as acetone or acetaldehyde where it is 3260 and $4890 \mathrm{~J} \mathrm{~mol}^{-1}$ [23]. The explanation lies probably in the existence of intramolecular hydrogen bond, which causes the shift of electron density from the carbonyl group to the O...H bond [23]. Taking the internal rotation constant $B_{\mathrm{CH} 3}$ of methyl group equal to $5.58 \mathrm{~cm}^{-1}$, with the barrier height $V_{3}$ equal to $67.5 \mathrm{~cm}^{-1}$, than $0 A \rightarrow 1 A$ transition of methyl group lies at $67.5 \mathrm{~cm}^{-1}$ (calculated by VIBAR [29]).

As far as $\mathrm{OH}$ torsion in the free molecule is concerned, Aspiala et al. [14] estimated from the ab initio calculations that the barrier would be around $3 \mathrm{~kJ} \mathrm{~mol}^{-1}\left(250 \mathrm{~cm}^{-1}\right)$. In the internal axis approximation, the $B_{\mathrm{OH}}$ is $16.18 \mathrm{~cm}^{-1}$; with the barrier height $V_{1}$ equal to $250 \mathrm{~cm}^{-1}$, the $0+\rightarrow 1+$ transition is calculated equal to $113.5 \mathrm{~cm}^{-1}$.

Since we do not have any spectra of pure hydroxyacetone, we compare these results with the information available for similar compounds and with the low frequency Raman spectra of water solution of hydroxyacetone (Fig. 6). At room temperature, the temperature reduced spectrum $R(\omega)$ is shown [30], while the spectrum at $10 \mathrm{~K}$ corresponds to a glassy state. In both cases there is a broad low frequency Raman mode at $\sim 80 \mathrm{~cm}^{-1}$. In ethanol, $\mathrm{OH}$ and $\mathrm{CH}_{2} \mathrm{OH}$ internal rotations are strongly coupled, and the two infrared vapour bands at 250 and $305.5 \mathrm{~cm}^{-1}$ interpreted as torsional transitions [31] and [32]. In ethylene glycol, $\mathrm{CH}_{2} \mathrm{OHCH}_{2} \mathrm{OH}$, there are three internal rotations present. Frei et al. observed $\mathrm{OH}$ torsions at 361 and $268 \mathrm{~cm}^{-1}$ in $\mathrm{Ar}$ matrix, and $\mathrm{CH}_{2} \mathrm{OH}$ torsion at $212 \mathrm{~cm}^{-1}$ in Xe matrix by infrared spectroscopy [33]. For the same molecule, Takeuchi and Tasumi observed infrared-induced rotamerization and assigned the $\mathrm{OH}$ torsional transitions to bands above $300 \mathrm{~cm}^{-1}$ while the $\mathrm{CH}_{2} \mathrm{OH}$ torsion was calculated to lie around $145 \mathrm{~cm}^{-1}$ for all conformers [25]. In glycolaldehyde the $\mathrm{OH}$ torsion is at $341 \mathrm{~cm}^{-1}$ [14] and the hydroxymethyl torsion at $195 \mathrm{~cm}^{-1}[22]$.

Bearing in mind the hydroxyl group dynamics in similar compounds discussed above, it seems probable that the broad low frequency Raman band at $80 \mathrm{~cm}^{-1}$ corresponds to $\mathrm{CH}_{2} \mathrm{OH}$ torsions in water solution of hydroxyacetone. As far as $\mathrm{OH}$ torsion is concerned, it is assigned to a weak $330 \mathrm{~cm}^{-1}$ Raman band which gains the intensity at $240 \mathrm{~K}$ and disappears on further cooling (see Fig. 7). The $\mathrm{CH}_{3}$ internal rotation transitions were not observed.

The unassigned 465 and $552 \mathrm{~cm}^{-1}$ Raman bands are probably skeletal bending bands (corresponding to OCC bending modes) of some other conformer. Also, there are two strong bands at 1083 and $1057 \mathrm{~cm}^{-1}$ in the infrared spectrum, corresponding to $\mathrm{C} \square \mathrm{O}$ stretching vibrations, supporting the hypothesis of at least two conformers existing in the solution. 


\section{Conclusion}

Vibrational assignment of hydroxyacetone was done by comparing the results of semi-empirical valence force field calculation (based on acetone, acetaldehyde and ethylene glycol and tested on glycoladehyde) with Raman and infrared bands of $90 \mathrm{wt} . \%$ water solution of hydroxyacetone. The $\mathrm{C} \square \mathrm{O}$ in plane bending mode was assigned to $615 \mathrm{~cm}^{-1}$ infrared and $617 \mathrm{~cm}^{-1}$ Raman band, the $\mathrm{C} \square \mathrm{O}$ out of plane mode to $503 \mathrm{~cm}^{-1}$ infrared and $504 \mathrm{~cm}^{-1}$ Raman band, the OCC bending to $276 \mathrm{~cm}^{-1}$ Raman band, and the CCC bending to $411 \mathrm{~cm}^{-1}$ Raman band. The hydroxymethyl group internal rotation transitions were observed in Raman spectrum of liquid and glass (below $150 \mathrm{~K}$ ) as a broad band centered around $80 \mathrm{~cm}^{-1}$, while the $\mathrm{OH}$ torsion is assigned to a weak and broad Raman band at $330 \mathrm{~cm}^{-1}$.

Two strong infrared bands at 1083 and $1057 \mathrm{~cm}^{-1}$ instead of one in the region of $\mathrm{C} \square \mathrm{O}$ stretching vibration indicate the presence of more than one conformer in the $90 \mathrm{wt} \%$ solution of hydroxyacetone in water. The shifting of the particularly temperature sensitive Raman band from 1053 to $1028.5 \mathrm{~cm}^{-1}$ on warming of the sample is explained as the change of hydrogen bonding in one of the confromers. The band at $1053 \mathrm{~cm}^{-1}$ is dominantly a $\mathrm{C} \square \mathrm{O}$ stretching mode, mixed with $\mathrm{COH}$ bending. It is via the bending mode that the hydrogen bonding manifests itself so sharply.

Also, in Raman spectrum there are two additional bands, at 465 and $552 \mathrm{~cm}^{-1}$, which are assigned to skeletal deformations of the second conformer. The NMR and UV results on diluted acetol state that hydroxyaceton is $96 \%$ in the carbonyl form and only up to $4 \%$ in the hydrated form [20]. The results presented here give evidence of at least two conformers of hydroxyacetone in the carbonyl form exist in the $90 \mathrm{wt} . \%$ water solution.

\section{Acknowledgements}

This work was supported by Ministry of Science and Technology of the Republic of Croatia, grant no. 0098019.

\section{References}

[1] B. Alberts, A. Johnson, J. Lewis, M. Raff, K. Roberts, P. Walter, Molecular Biology of the Cell, Garland Publication, 2002.

[2] G.M. Fuller, D. Shields, Molecular Basis of Medical Cell Biology, Appleton \& Lange, Stamford, CO, 1998.

[3] L. Stryer, Biochemistry, W.H. Freeman \& Co, New York, 1988.

[4] R.V. Iozzo, Annu. Rev. Biochem. 67 (1998) 609.

[5] E.A. Balasz (Ed.), Chemistry and Molecular Biology of the Intercellular Matrix, vols. 1-3, Academic Press, 1970.

[6] L. Penov, A.F. Beema, Free Rad. Biol. Med. 34 (4) (2003) 429. 
Mohaček-Grošev, V., (2005), "Vibrational analysis of hydroxyacetone" Spectrochimica Acta Part A: Molecular and Biomolecular Spectroscopy, Vol. 61, No. 3, pp. 477-484.

[7] P.E. Morgan, R.T. Dean, M.J.J. Davies, Arch. Biochem. Biophys. 403 (2002) 259.

[8] D. Ruggiero-Lopez, M. Lecomte, G. Moinet, G. Patereau, M. Lagarde, N. Wiernsperger, Biochem. Pharmacol. 58 (1999) 1765.

[9] G.K. Glushonok, E.P. Petrayev, O.I. Shadyro, Zh. Fiz. Khim. 58 (1984) 111.

[10] G.K. Glushonok, E.P. Petrayev, E.A. Turetskaya, O.I. Shadyro, Zh. Fiz. Khim. 60 (1986) 2960.

[11] G.K. Glushonok, T.G. Glushonok, O.I. Shadyro, Kinet. Catal. 41 (5) (2000) 620.

[12] Y. Kobayashi, H. Takahara, H. Takahashi, K. Higasi, J. Mol. Struct. 32 (1976) 235.

[13] H. Michelsen, P. Klaboe, J. Mol. Struct. 4 (1969) 293.

[14] A. Aspiala, J. Murto, P. Sten, Chem. Phys. 106 (1986) 399.

[15] V.A. Yaylayan, S. Harty-Majors, A.A. Ismail, Carbohydr. Res. 309 (1998) 31.

[16] D. Gardiner, Carbohydr. Res. 2 (1966) 234.

[17] G.C.S. Collins, W.O. George, J. Chem. Soc. B (1971) 1352.

[18] C.I. Stassinopoulou, C. Zioudrou, Tetrahedron 28 (1972) 1257.

[19] Y. Kobayashi, H. Takahashi, Spectrochim. Acta 35A (1979) 307.

[20] G.K. Glushonok, T.G. Glushonok, I.P. Edimencheva, O.I. Shadyro, High Energy Chem. 33 (6) (1999) 364.

[21] K.M. Marstokk, H. Møllendal, J. Mol. Struct. 5 (1970) 205.

[22] K.M. Marstokk, H. Møllendal, J. Mol. Struct. 16 (1973) 259.

[23] M. Kattija-Ari, M.D. Harmony, Int. J. Quant. Chem. Quant. Chem. Symp. 14 (1980) 443453.

[24] P. Cossee, J.H. Schachtschneider, J. Chem. Phys. 44 (1) (1966) 97.

[25] H. Takeuchi, M. Tasumi, Chem. Phys. 77 (1983) 21.

[26] J.H. Schachtschneider, F.S. Martiner, Technical Report Nos. 57-65 and 231-264, Shell, Emeryville, CA, 1964.

[27] G. Bouchoux, F. Penaud-Berruyer, W. Bertrand, Eur. J. Mass Spectrom. 7 (2001) 351.

[28] Yong Wu, Daiqian Xie Xie, Ying Xue, J. Comput. Chem. 24 (2003) 931.

[29] J.R. Durig, S.M. Craven,W.C. Harris, in: J.R. Durig (Ed.), Vibrational Spectra and

Structure, vol. 1, Marcel Dekker Inc., New York, Chapter 4, 1972.

[30] M.H. Brooker, O. Faurskar Nielsen, E. Praestgaard, J. Raman Spectrosc. 19 (1988) 71.

[31] J.R. Durig, R.A. Larsen, J. Mol. Struct. 238 (1989) 195.

[32] M.L. Senent, Y.G. Smeyers, R. Dominguez-Gomez, M. Villa, J. Chem. Phys. 112 (2000) 5809.

[33] H. Frei, T.-K. Ha, R. Meyer, Hs.H. Gunthard, Chem. Phys. 25 (1977) 271.

[34] V. Moha cek-Grošev, F. Stelzer, D. Jocham, J. Mol. Struct. 476 (1999) 181. 